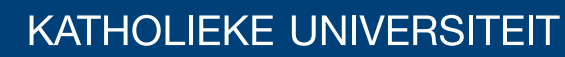 \\ LEUVEN
}

\section{Faculty of Business and Economics}

Robust regression in Stata

V. Verardi and C. Croux

DEPARTMENT OF DECISION SCIENCES AND INFORMATION MANAGEMENT (KBI) 


\title{
Robust Regression in Stata
}

\author{
Vincenzo Verardi* and Christophe Croux !
}

\begin{abstract}
In regression analysis, the presence of outliers in the data set can strongly distort the classical least squares estimator and lead to unreliable results. To deal with this, several robust-to-outliers methods have been proposed in the statistical literature. In Stata, some of these methods are available through the commands rreg and qreg. Unfortunately, these methods only resist to some specific types of outliers and turn out to be ineffective under alternative scenarios. In this paper we present more effective robust estimators that we implemented in Stata. We also present a graphical tool that allows recognizing the type of existing outliers.
\end{abstract}

KEYWORDS: S-estimators, MM-estimators, Outliers, Robustness

JEL CLASSIFICATION: C12, C21, C87.

\section{Introduction}

The objective of linear regression analysis is to study how a dependent variable is linearly related to a set of regressors. In matrix notation, the linear regression model is given by:

$$
y=X \theta+\varepsilon
$$

where, for a sample of size $n, y$ is the $(n \times 1)$ vector containing the values for the dependent variable, $X$ is the $(n \times p)$ matrix containing the values for the $p$ regressors and $\varepsilon$ is the $(n \times 1)$ vector containing the error terms. The $(p \times 1)$ vector $\theta$ contains the unknown regression parameters and needs to be estimated. On the basis of the estimated parameter $\hat{\theta}$, it is then possible to fit the dependent variable by $\hat{y}=X \hat{\theta}$, and estimate the residuals $r_{i}=y_{i}-\hat{y}_{i}$, for $i=1 \leq i \leq n$. Although $\theta$ can be estimated in several ways, the underlying idea is always to try to get as close as possible to the true value by reducing the magnitude of the residuals, as measured by an aggregate prediction error. In the case of the well-known ordinary least squares (LS), this aggregate prediction error is defined as the sum of squared residuals. The vector of parameters estimated by LS is then

\footnotetext{
*University of Namur (CRED) and Université Libre de Bruxelles (ECARES and CKE). Rempart de la Vierge, 8. B-5000 Namur. E-mail: vverardi@fundp.ac.be. Vincenzo Verardi is Associated Researcher of the FNRS and gratefully acknowledges their financial support.

${ }^{\dagger}$ K.U.Leuven, Faculty of Business and Economics, Naamsestraat $69 . \quad$ B-3000, Leuven. Email: christophe.croux@econ.kuleuven.be.
} 


$$
\hat{\theta}_{L S}=\arg \min _{\theta} \sum_{i=1}^{n} r_{i}^{2}(\theta)
$$

with $r_{i}(\theta)=y_{i}-\theta_{0}-\theta_{1} X_{i 1}-\ldots-\theta_{1} X_{i p}$ for $1 \leq i \leq n$. This estimation can be performed in Stata using the regress command. A drawback of LS is that, by considering squared residuals, it tends to award an excessive importance to observations with very large residuals and, consequently, distort parameters' estimation in case of existence of outliers.

The scope of this paper is first, to describe regression estimators that are robust with respect to outliers and, second, to propose Stata commands to implement them in practice. The structure of the paper is the following: we briefly present, in Section 2, the type of outliers that can be found in regression analysis and introduce the basics of robust regression. We recommend to use high-breakdown point estimators (i.e. estimators that resist to a contamination of up to $50 \%$ of the observations), which are known to be resistant to outliers of different types. In Section 3, we describe them and provide a sketch of the Stata code we implemented to estimate them in practice. In Section 4 we give an example using the well-known Stata auto dataset. In Section 5 we provide some simulation results to illustrate how the high-breakdown point estimators outperform the robust estimators available in Stata. Finally, in Section 6 we conclude.

\section{Outliers and robust regression estimators}

In regression analysis, three types of outliers influence the LS estimator. Rousseeuw and Leroy (1987) define them as vertical outliers, bad leverage points and good leverage points. To illustrate this terminology, consider a simple linear regression as shown in Figure 1 (the generalization to higher dimensions is straightforward). Vertical outliers are those observations that have outlying values for the corresponding error term (the $y$-dimension) but are not outlying in the space of explanatory variables (the $x$-dimension). Their presence affects the LS-estimation and in particular the estimated intercept. Good Leverage points are observations that are outlying in the space of explanatory variables but that are located close to the regression line. Their presence does not affect the LS-estimation but it affects statistical inference since they do inflate the estimated standard errors. Finally, Bad Leverage points are observations that are both outlying in the space of explanatory variables and located far from the true regression line. Their presence affects significantly the LS-estimation of both the intercept and the slope.

Edgeworth (1887) realized that due to the squaring of the residuals, LS becomes extremely vulnerable to the presence of outliers. To cope with this, he proposed a method consisting in minimizing the sum of the absolute values of the residuals rather than the sum of their squares. More precisely, his method 


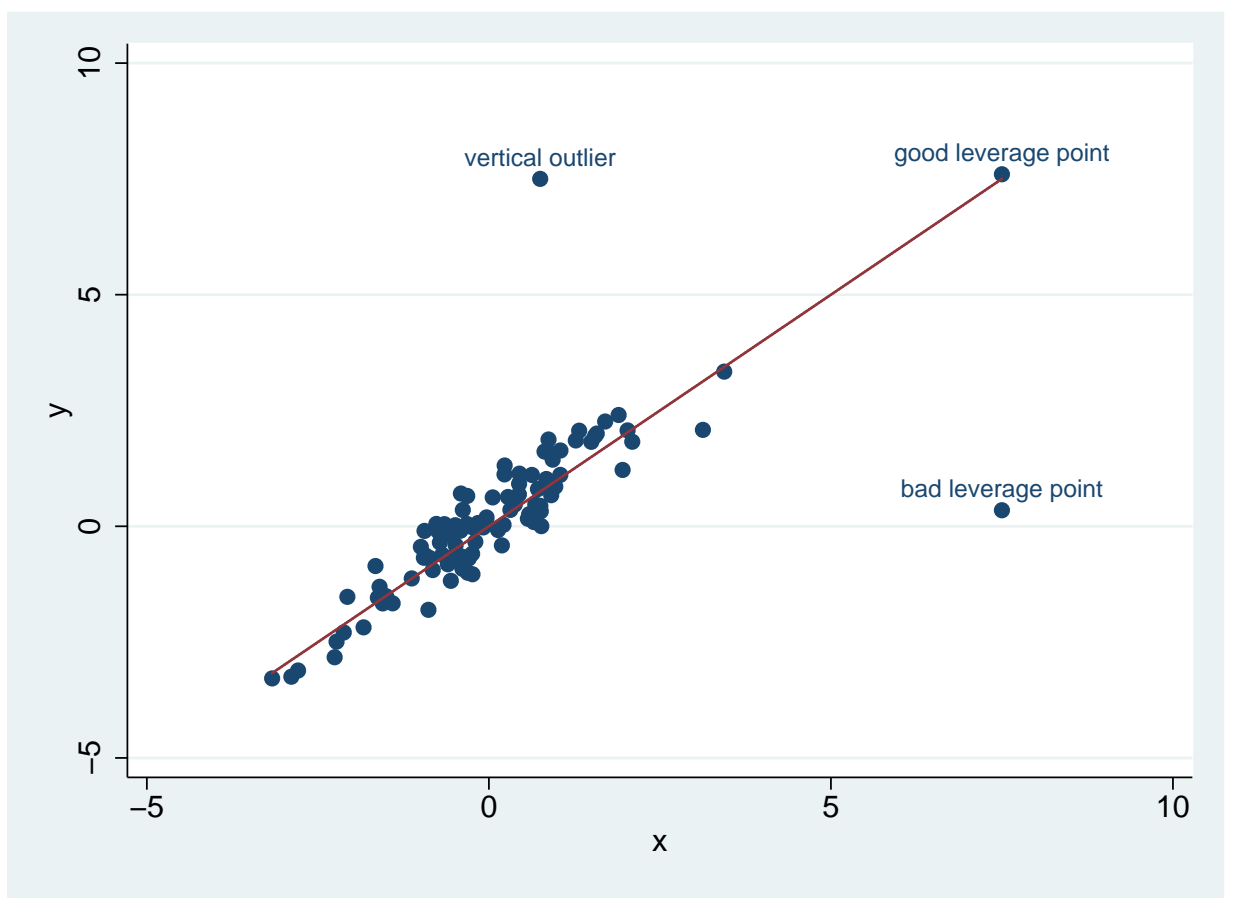

Figure 1: Outliers in regresson analysis

defines the $L_{1}$ or median regression estimator as

$$
\hat{\theta}_{L_{1}}=\arg \min _{\theta} \sum_{i=1}^{n}\left|r_{i}(\theta)\right|
$$

The median regression estimator is available in Stata via the qreg command as a standard function. This estimator does protect against vertical outliers but not against bad leverage points. It has an efficiency of only $64 \%$ at a Gaussian error distribution (see Huber, 1981).

Huber (1964) generalized median regression to a wider class of estimators, called M-estimators, by considering other functions than the absolute value in (3). This allows to increase Gaussian efficiency while keeping robustness with respect to vertical outliers. An M-estimator is defined as

$$
\hat{\theta}_{M}=\arg \min _{\theta} \sum_{i=1}^{n} \rho\left(\frac{r_{i}(\theta)}{\sigma}\right)
$$

where $\rho(\cdot)$ is a loss function which is even, non decreasing for positive values and less increasing than the square function. To guarantee scale equivariance (i.e. independence with respect to the measurement units of the dependent variable), residuals are standardized by a measure of dispersion $\sigma$. M-estimators are called monotone if $\rho(\cdot)$ is convex over the entire domain and redescending if $\rho(\cdot)$ is bounded ${ }^{1}$.

\footnotetext{
${ }^{1}$ This terminology is related to $\rho^{\prime}$.
} 
The practical implementation of M-estimators uses an iteratively reweighted least squares algorithm. To simplify, suppose that $\sigma$ is known and define weights $\omega_{i}=\rho\left(r_{i} / \sigma\right) / r_{i}^{2}$, then equation (4) can be rewritten as

$$
\hat{\theta}_{M}=\arg \min _{\theta} \sum_{i=1}^{n} \omega_{i} r_{i}^{2}(\theta),
$$

which is a weighted least-squares estimator. The weights $\omega_{i}$ are however a function of $\theta$ and are thus unknown. Using an initial estimate $\tilde{\theta}$ for $\theta$, the weights can be computed and serve as the start of an iteratively reweighted least squares algorithm. Unfortunately, the latter is guaranteed to converge to the global minimum of (4) only for monotone M-estimators which are not robust with respect to bad leverage points.

In Stata, the rreg command computes a highly efficient M-estimator. The loss function used is a Tukey Biweight function defined as

$$
\rho(u)= \begin{cases}1-\left[1-\left(\frac{u}{k}\right)^{2}\right]^{3} & \text { if }|u| \leq k \\ 1 & \text { if }|u|>k\end{cases}
$$

where $k=4.685$. The starting value of the iterative algorithm $\tilde{\theta}$ is taken to be a monotone M-estimator with a Huber $\rho(\cdot)$ function:

$$
\rho(u)= \begin{cases}\frac{1}{2}(u)^{2} & \text { if }|u| \leq c \\ c|u|-\frac{1}{2} c^{2} & \text { if }|u|>c\end{cases}
$$

where $c=1.345$. Moreover, to give protection against bad leverage points, observations associated to Cook distances larger than 1 , receive a weight zero.

Unfortunately, the rreg command has not the expected robustness properties for two main reasons. First, Cook distances only manage to identify isolated outliers and are inappropriate in case of existence of clusters of outliers, where one outlier can mask the presence of another (see Rousseeuw and Van Zomeren, 1990). It can therefore not be guaranteed to have identification of all leverage points. Second, the initial values for the iteratively reweighted least squares algorithm are monotone M-estimators that are not robust to bad leverage points and may lead the algorithm to converge to a local instead of a global minimum.

\section{High Break-down point estimators}

Full robustness can be achieved by tackling the regression problem from a different perspective. Recall that the LS estimator is based on the minimization of the variance of the residuals. Hence, since the 
variance is highly sensitive to outliers, LS is largely influenced as well. For this reason, Rousseeuw and Yohai (1987) propose to minimize a measure of dispersion of the residuals that is less sensitive to extreme values than the variance ${ }^{2}$. They call this class of estimators the S-estimators. The intuition behind the method is simple. For LS, the objective is to minimize the variance $\hat{\sigma}^{2}$ of the residuals. The latter can be rewritten as $\frac{1}{n} \sum_{i=1}^{n}\left(\frac{r_{i}}{\hat{\sigma}}\right)^{2}=1$. As stated previously, the square value can be damaging as it gives a huge importance to large residuals. Thus, to increase robustness, the square function could be replaced by another loss function $\rho$ which awards less importance to large residuals ${ }^{3}$. The estimation problem would now consist in finding the smallest robust scale of the residuals. This robust dispersion, that will be called $\hat{\sigma}^{S}$ satisfies

$$
\frac{1}{n} \sum_{i=1}^{n} \rho\left(\frac{r_{i}(\theta)}{\hat{\sigma}^{S}}\right)=b
$$

where $b=E[\rho(Z)]$ with $Z \sim N(0,1)$. The value of $\theta$ that minimizes $\hat{\sigma}^{S}$ is then called an S-estimator. More formally, an S-estimator is defined as:

$$
\hat{\theta}^{S}=\arg \min _{\theta} \hat{\sigma}^{S}\left(r_{1}(\theta), \ldots, r_{n}(\theta)\right)
$$

where $\hat{\sigma}^{S}$ is the robust estimator of scale as defined in (8).

The choice of $\rho(\cdot)$ is crucial to have good robustness properties and a high Gaussian efficiency. The Tukey Biweight function defined in (6), with $k=1.547$, is a common choice. This S-estimator resists to a contamination of up-to $50 \%$ of outliers. In other words, it is said to have a breakdown point of $50 \%$. Unfortunately, this S-estimator has a Gaussian efficiency of only $28.7 \%$. If $k=5.182$, the Gaussian efficiency raises to $96.6 \%$ but the breakdown point drops to $10 \%$. To cope with this, Yohai (1987) introduced MM-estimators that combine high-breakdown point and a high efficiency. These estimators are redescending M-estimators as defined in (4), but where the scale is fixed at $\hat{\sigma}^{S}$. So an MM-estimator is defined as

$$
\hat{\theta}^{M M}=\arg \min _{\theta} \sum_{i=1}^{n} \rho\left(\frac{r_{i}(\theta)}{\hat{\sigma}^{S}}\right)
$$

The preliminary S-estimator guarantees a high breakdown point, and the final MM-estimate allows a high Gaussian efficiency. It is common to use a Tukey Biweight $\rho(\cdot)$ function for both the preliminary S-estimator and the final MM-estimator. The tuning constant $k$ can be set to 1.547 for the S-estimator,

\footnotetext{
${ }^{2}$ Note that the Least Trimmed Squares estimator and the Least Median Squares estimator, introduced by Rousseeuw (1984) rely on the same logic. We programmed these two estimators in Stata, and made available through the command ltsregress and lmsregress.

${ }^{3}$ As before, $\rho(\cdot)$ is a function which is even, non decreasing for positive values, less increasing than the square with a unique minimum at zero
} 
to guarantee a $50 \%$ breakdown point, and it can be set to 4.685 for the second step MM-estimator in (10) to guarantee a $95 \%$ efficiency of the final estimator. If $k=2.697$ for the second step, the efficiency of the MM-estimator will be $70 \%$. For computing the MM-estimator, the iteratively reweighted least squares algorithm can be used, taking $\hat{\theta}^{S}$ as initial value. Once the initial S-estimate is computed, $\hat{\theta}^{M M}$ comes at almost no additional computational cost. We programmed an S- and an MM-estimator in Stata (with Tukey Biweight loss function) using the algorithm of Salibian-Barrera and Yohai (2006). We present in the next section a sketch of the algorithm.

\subsection{S-estimator and MM-estimator algorithm}

The algorithm implemented in Stata for computing the S-estimator starts by randomly picking $N$ subsets of $p$ observation (defined as $p$-subset) where $p$ is the number of regression parameters to estimate. For each $p$-subset, the equation of the hyperplane that fits all points perfectly is obtained yielding a trial solution of (9). This trial value is more reliable if all $p$ points are regular observations, such that the $p$-subset does not contain outliers. The number $N$ of sub-samples to generate is chosen to guarantee that at least one $p$-subset without outliers is selected with high probability. As shown in Salibian-Barrera and Yohai (2006), this can be achieved by taking

$$
N=\left\lceil\frac{\log \left(1-P_{\text {clean }}\right)}{\log \left[1-(1-\alpha)^{p}\right]}\right\rceil
$$

where $\alpha$ is the (maximal) expected proportion of outliers, $p$ is the number of parameters to estimate and $P_{\text {clean }}$ is the desired probability of having at least one $p$-subset without outliers among the $N$ subsamples ${ }^{4}$.

For each of the $p$-subsets, residuals are computed as the vertical distance separating each observation from the hyperplane that perfectly fits the $p$-subset. On the basis of these residuals, a scale estimate $\hat{\sigma}^{S}$ is obtained as in (8) and this for each $p$-subset. An approximation for the final scale estimate $\hat{\sigma}^{S}$ is then given by the trial value that leads to the smallest scale over all p-subsets. This approximation can be improved further by carrying some refinement steps, that bring the approximation even closer to the solution of $(9)$.

This algorithm is implemented in Stata and can be called using the MMregress function and invoking the initial option. Once the S-estimator is obtained, the MM-estimator directly follows by applying the iteratively reweighted least squares algorithm up to convergence. We provide a Stata command (MMregress) to estimate a wide range of MM-estimators. It is described in details in section 6 . As far as inference is concerned, standard errors robust to heteroskedasticity are computed according to the formulas available in the literature (see e.g. Croux, Dhaene and Horelbeke, 2008).

\footnotetext{
${ }^{4}$ The default values we use in the implementation of the algorithm are $\alpha=0.2$ and $P_{\text {clean }}=0.99$.
} 
The need of calling on subsampling algorithms becomes Achille's heel of the algorithm when several dummy variables are present. Indeed, as stated by Maronna and Yohai (2000), subsampling algorithms can easily lead to collinear sub-samples if various dummies are among the regressors. To cope with this, Maronna and Yohai (2000) introduce the MS-estimator that alternates an S-estimator (for continuous variables) and an M-estimator (for dummy ones), till convergence. This estimator is somehow out of the scope of the paper and we thus do not elaborate on it here. It is however important to state that we implemented this estimator in Stata and we can call it with the function MMregress invoking the appropriate options (i.e. dummies and initial). This estimator can be particularly helpful in the fixed effects panel data models, as suggested by Bramati and Croux (2007).

\subsection{Outlier detection}

An important think to stress is that, in addition to reducing the importance of outliers on the estimator, robust statistics are also intended to identify atypical individuals. Once identified, they could be analyzes separately from the bulk of data. Tho do so, it is important to recognize their type. This can be easily achieved by calling on the graphical tool proposed by Rousseeuw and Van Zomeren (1990). This graphical tool is constructed by plotting on the vertical axis the Robust Standardized Residuals, defined as $r_{i} / \hat{\sigma}^{S}$, with $r_{i} \equiv r_{i}\left(\hat{\theta}^{S}\right)$, to give an idea of outlyingness with respect to the fitted regression plane. On the horizontal axis a measure of the (multivariate) outlyingness of the explanatory variables is plotted. The latter is measured by the Mahalanobis distance defined as $d_{i}=\sqrt{\left(X_{i}-\mu\right) \Sigma^{-1}\left(X_{i}-\mu\right)^{\prime}}$ where $\mu$ is the multivariate location vector, $\Sigma$ is the covariance matrix of the explanatory variables and $X_{i}$ the $i^{\text {th }}$ row-vector of matrix $X$, for $i \leq i \leq n$. Obviously both $\mu$ and $\Sigma$ should be estimated robustly if we want these distances to resist to the presence of outliers. Several methods have been proposed to estimate robustly the Mahalanobis distances. In Stata, the command hadimvo is available but, more robust estimates for the covariance matrix (such as the Minimum Covariance Determinant estimator) are also available.

It is then possible to set the limits outside which individuals can be considered as outliers. For the $y$-dimension, we set them to -2.25 and +2.25 . These represent the values of the Standard Normal that separate the $2.5 \%$ remotest area of the distribution from the central mass. For the $x$-dimension we set the limit to $\sqrt{\chi_{p, 0.975}^{2}}$, motivated by the fact that the squared Mahalanobis distance is distributed as a $\chi_{p}^{2}$ distribution under normality. 


\section{Example}

To illustrate the usefulness of the robust methods, we present an example based on the well-known stata auto.dta dataset. More specifically, we regress the price of cars on the following set of characteristics: the mileage, the headroom, the trunk space, the weight, the length, the turn circle, the displacement, the gear ratio, four dummies identifying the categorical variable repair record in 1978, and a foreign dummy identifying if the car is not built in the US. The first thing we do is identify outliers. For this purpose we call on the graphical tool described in Section 3.2. The resulting plot is pictured in Figure 2. Several features emerge. First, the Cadillac Seville is a bad leverage point. Indeed it is an outlier in the horizontal as well as in the vertical dimension. This means that its characteristics are pretty different from those of the bulk of data and its price is much higher than it should be according to the fitted model. The Volkswagen Diesel and the Plymouth Arrow are large good leverage points since they are outlying in the horizontal dimension but not on the vertical one. This means that their characteristics are rather different from the other cars however their price is in accordance with what the model predicts. Finally the Cadillac Eldorado, the Lincoln Versaille, the Lincoln Mark V, the Volvo 260 and some others are standard in their characteristics but are more expensive than the model would suggest. They correspond to vertical outliers.

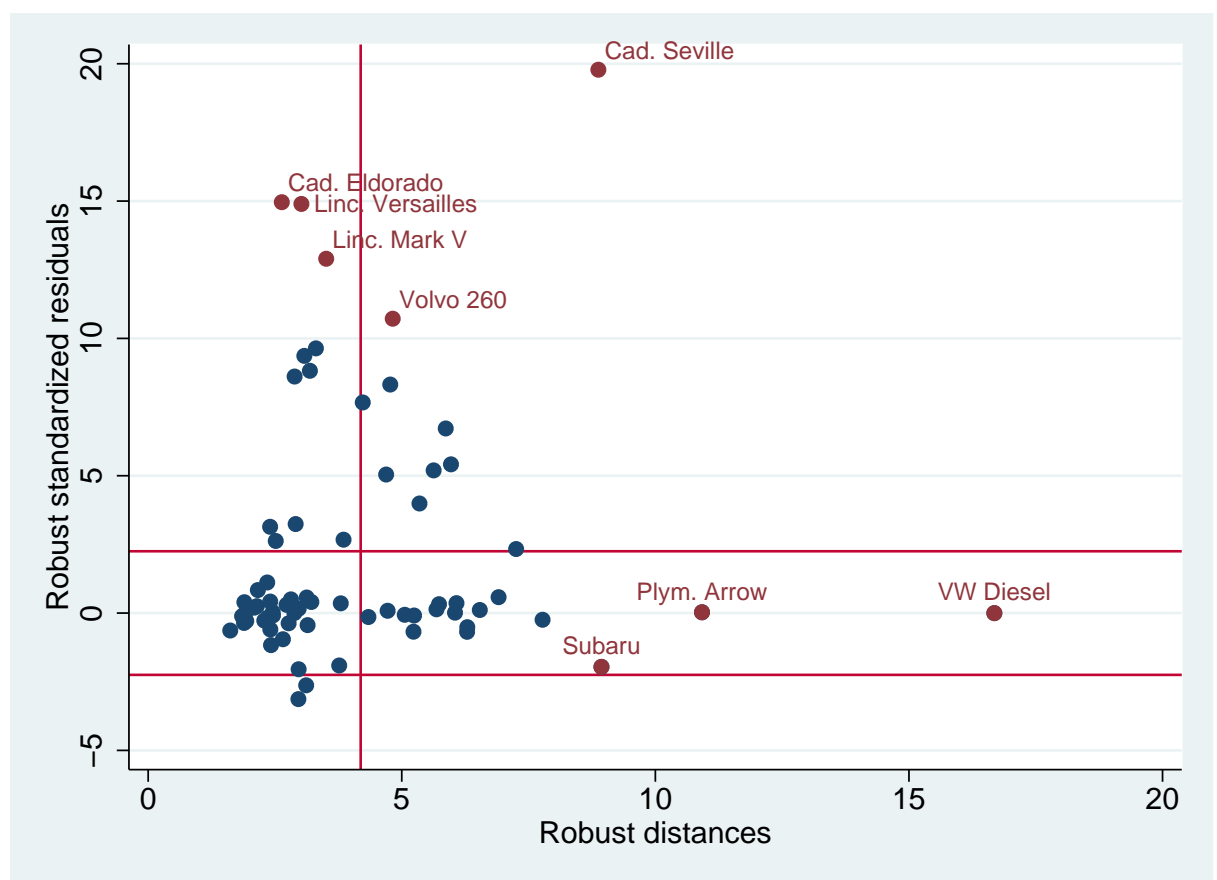

Figure 2: Regression outliers in the "auto.dta" Stata dataset 
Are these outlying observations sufficient to distort classical estimations? Since several vertical outliers are present as well as a severe bad leverage point, there is a serious risk that the least squares estimator becomes strongly attracted by the outliers. To illustrate this, we compare the results obtained using the recommended high breakdown point estimator MMregress with those obtained using regress, M-estimator (rreg) and median regression (qreg). The differences are, as expected, important. We present the results in Table 1.

Table 1: Determinants of car prices in the "auto.dta" Stata dataset.

\begin{tabular}{|c|c|c|c|c|c|}
\hline Dependent: Price in US\$ & regress & qreg & rreg & $\operatorname{MM}(0.70)$ & $\operatorname{MM}(0.95)$ \\
\hline Mileage (mpg) & $\begin{array}{c}-43.95 \\
(0.52)\end{array}$ & $\begin{array}{c}-44.45 \\
(0.55)\end{array}$ & $\begin{array}{r}-68.91 \\
(0.92)\end{array}$ & -44.88 & $\begin{array}{c}-46.74 \\
(1.56)\end{array}$ \\
\hline Headroom (in.) & $-\underset{(1.72)}{689.40^{*}}$ & $-\underset{(1.71)}{624.19^{*}}$ & $\begin{array}{c}-739.30^{* *} \\
(2.09)\end{array}$ & $-311.96^{* *}$ & $-440.06^{* * *}$ \\
\hline Trunk space (cu. ft.) & $\begin{array}{l}74.29 \\
(0.74)\end{array}$ & $\begin{array}{l}37.50 \\
(0.40)\end{array}$ & $\begin{array}{c}114.53 \\
(1.29)\end{array}$ & $\begin{array}{c}186.60^{* * *} \\
(7.10)\end{array}$ & $\begin{array}{c}128.98^{* * *} \\
(3.53)\end{array}$ \\
\hline Weight (lbs.) & $\begin{array}{l}4.67^{* * *} \\
(3.19)\end{array}$ & $\begin{array}{c}2.89^{* *} \\
(2.10)\end{array}$ & $\begin{array}{l}2.59^{*} \\
(1.99)\end{array}$ & $\underset{(5.29)}{1.03^{* * *}}$ & $\begin{array}{l}0.37 \\
(0.62)\end{array}$ \\
\hline Length (in.) & $-\underset{(1.86)}{80.66^{*}}$ & $\begin{array}{r}-48.78 \\
(1.17)\end{array}$ & $\begin{array}{c}-27.50 \\
(0.72)\end{array}$ & $-33.74^{* *}$ & $\begin{array}{l}0.03 \\
(0.00)\end{array}$ \\
\hline Turn Circle (ft.) & $\begin{array}{c}-143.71 \\
(1.11)\end{array}$ & $\begin{array}{l}30.22 \\
(0.30)\end{array}$ & $\begin{array}{c}-104.26 \\
(0.91)\end{array}$ & $\begin{array}{l}10.51 \\
(0.48)\end{array}$ & $\begin{array}{c}-23.79 \\
(0.69)\end{array}$ \\
\hline Displacement (cu. in.) & $\begin{array}{l}12.71 \\
(1.45)\end{array}$ & $\begin{array}{l}9.79 \\
(1.27)\end{array}$ & $\begin{array}{l}11.34 \\
(1.46)\end{array}$ & $\begin{array}{l}2.31 \\
(0.98)\end{array}$ & $\begin{array}{l}2.51 \\
(0.58)\end{array}$ \\
\hline Gear Ratio & $\underset{(0.09)}{115.08}$ & $\begin{array}{l}92.28 \\
(0.08)\end{array}$ & $\underset{(0.82)}{917.19}$ & $\begin{array}{c}492.467 \\
(0.89)\end{array}$ & $\begin{array}{c}370.20 \\
(0.99)\end{array}$ \\
\hline Foreign & $\begin{array}{c}3064.52^{* * *} \\
(2.89)\end{array}$ & $\underset{(2.38)}{2496.04^{* *}}$ & $\underset{(2.48)}{2326.91^{* *}}$ & $\begin{array}{c}-91.66 \\
(0.19)\end{array}$ & $\begin{array}{c}763.91^{*} \\
(1.89)\end{array}$ \\
\hline rep $78==2$ & $\begin{array}{c}1353.80 \\
(0.79)\end{array}$ & $\begin{array}{c}-355.92 \\
(0.27)\end{array}$ & $\begin{array}{c}465.98 \\
(0.31)\end{array}$ & $\begin{array}{l}5.99 \\
(0.02)\end{array}$ & $\begin{array}{l}31.45 \\
(0.11)\end{array}$ \\
\hline $\operatorname{rep} 78==3$ & $\begin{array}{c}955.44 \\
(0.59)\end{array}$ & $\begin{array}{l}19.24 \\
(0.02)\end{array}$ & $\begin{array}{c}488.23 \\
(0.34)\end{array}$ & $-720.50^{* * *}$ & $-\underset{(1.17)}{286.70}$ \\
\hline rep $78==4$ & $\begin{array}{c}976.63 \\
(0.59)\end{array}$ & $\begin{array}{c}241.79 \\
(0.18)\end{array}$ & $\underset{(0.55)}{813.11}$ & $\begin{array}{c}-275.89 \\
(1.04)\end{array}$ & $\underset{(1.49)}{390.71}$ \\
\hline $\operatorname{rep} 78==5$ & $\begin{array}{c}1758.00 \\
(0.97)\end{array}$ & $\underset{(0.91)}{1325.18}$ & $\underset{(0.95)}{1514.13}$ & $\begin{array}{c}606.77^{*} \\
(1.70)\end{array}$ & $\begin{array}{c}359.01 \\
(0.86)\end{array}$ \\
\hline Constant & $\begin{array}{c}9969.75 \\
(1.40)\end{array}$ & $\begin{array}{c}4083.51 \\
(0.60)\end{array}$ & $\begin{array}{c}2960.68 \\
(0.47)\end{array}$ & $\begin{array}{c}5352.18^{* * *} \\
(3.10)\end{array}$ & $\begin{array}{c}3495.97 \\
(1.43)\end{array}$ \\
\hline
\end{tabular}

Absolute value of $\mathrm{t}$ statistics in parentheses.

Significant at $* * * 1 \%, * * 5 \%, * 10 \%$

Let's compare the results. First the mileage, headroom, trunk space and length seem to be unimportant in explaining prices (at a $5 \%$ level) when looking at the OLS, median and M-estimators (i.e. regress, qreg and rreg). However, when the influence of outliers (and especially of the bad leverage point) is taken into account (i.e. MM(0.7) column), they turn out to be significantly different to zero. If we consider a more efficient estimator (i.e. MM(0.95) column) weight and length become again insignificant. The weight variable is flagged as significant by most specifications (though the size of 
effect is very different). The turn, displacement and gear ratio variables turn out to be insignificant in all specifications. The foreign dummy is insignificant only using the most robust estimators.

\section{Simulations}

Several recent articles have proven the theoretical properties of the estimators described in the previous sections. In this paper we will compare the performances of the Stata codes we implemented with the previously available robust commands and LS. To do so we run some simulations according to the following setup. We start by creating a dataset (of size $n=1000$ ) by randomly generating 5 independent explanatory continuous variables (labelled $\left.X_{1}, \ldots, X_{5}\right)$ and an error term $(e)$ from six independent univariate normal distributions with mean zero and unit variance. A $y$ variable is then generated according to the formula $y_{i}=\beta_{0}+\sum_{j=1}^{5} \beta_{j} X_{i j}+e_{i}$ where $\beta_{0}=0$ and $\beta_{j}=1$ for $j=1, \ldots, 5$. This dataset is called the clean dataset. We then contaminate the data by replacing randomly $10 \%$ of the $X_{1}$ observations without modifying $y$. These contaminated points are generated from a normal distribution with mean 5 and standard deviation 0.1 and are bad leverage points. We call this the contaminated dataset. We then repeat this procedure 1000 times and each time we estimate the parameters using LS, $\mathrm{L}_{1}, \mathrm{M}, \mathrm{S}$ and MM-estimators (with a $95 \%$ and a $70 \%$ efficiency). On the basis of all the estimated parameters, we measure the bias (i.e. the average of the estimated parameters minus the true value) and the mean squared error (i.e. the variance of the estimated parameters plus the square of the bias). The results are presented in Table 2. We do not present the results associated to the clean sample since all estimation methods lead to comparable outcomes and very low biases. 
Table 2: Simulated Bias and MSE of LS, $\mathrm{L}_{1}, \mathrm{M}$ and MM-estimators

\begin{tabular}{lccccccc}
\hline \hline Estimation method & & $\beta_{1}$ & $\beta_{2}$ & $\beta_{3}$ & $\beta_{4}$ & $\beta_{5}$ & $\beta_{0}$ \\
\hline LS (reg) & Bias & 0.7149 & 0.0015 & 0.0010 & 0.0002 & 0.0016 & -0.1440 \\
& MSE & 0.5118 & 0.0017 & 0.0018 & 0.0019 & 0.0018 & 0.0223 \\
\hline L $_{1}$ (qreg) & Bias & 0.6369 & 0.0006 & 0.0013 & 0.0004 & 0.0011 & -0.1281 \\
& MSE & 0.4071 & 0.0026 & 0.0024 & 0.0027 & 0.0027 & 0.0188 \\
\hline M (rreg) & Bias & 0.6725 & 0.0012 & 0.0010 & 0.0005 & 0.00167 & -0.1353 \\
& MSE & 0.4532 & 0.0018 & 0.0018 & 0.0019 & 0.0019 & 0.0200 \\
\hline MM (MMregress, eff(0.95)) & Bias & 0.6547 & 0.0011 & 0.0009 & 0.0010 & 0.00167 & -0.1318 \\
& MSE & 0.4298 & 0.0018 & 0.0018 & 0.0020 & 0.0020 & 0.0190 \\
\hline MM (MMregress, eff(0.70)) & Bias & 0.0867 & 0.0012 & 0.0028 & -0.0008 & -0.0010 & -0.0164 \\
& MSE & 0.0236 & 0.0015 & 0.0015 & 0.0015 & 0.0014 & 0.0024 \\
\hline
\end{tabular}

Simulation setup: sample size $=1000$, simulations $=1000$, contamination $=10 \%$.

The results of the simulations clearly show that, for this contamination setup, the less biased estimator among those we considered is the MM-estimator with an efficiency of $70 \%$. Its bias and MSE are respectively of 0.087 and 0.024 for $\beta_{1}$ and of -0.016 and 0.002 for $\beta_{0}$. As a comparison, the bias and MSE of LS are 0.715 and 0.512 for $\beta_{1}$ and -1.144 and 0.02 for $\beta_{0}$. For the other coefficients the performances of all estimators are comparable. It is important to stress that if we set the efficiency of $\mathrm{MM}$ to $95 \%$, its performance in terms of bias worsens too much and would thus not be desirable. The $\mathrm{L}_{1}$ and M-estimators (computed respectively with the qreg and rreg commands) behave rather poorly and have a bias and an MSE comparable to that of LS.

\section{The MMregress command}

The MMregress command computes the high breakdown point regression estimators, described in Section 3, and their standard errors. The general syntax for the command is:

MMregress varlist [if exp] [in range] [, eff(\#) dummies (varlist) noconstant outlier graph replic(\#) init]

The first optional parameter is eff, which allows to fix the efficiency of the MM-estimator. It can take any value between 0.287 and 1 ; the higher its value, the more efficient the MM-estimator. While the breakdown point of the MM-estimator is always $50 \%$, its bias increases with its efficiency. Therefore, to have a good compromise between robustness and efficiency of the MM-estimator, we take 
as a default value eff $=70 \%$. The dummies option allows to declare which variables are dichotomous. In case dummies is declared, the initial estimator will be the MS rather than the S-estimator. Not declaring this option when dummy variables are present may cause the algorithm for computing the S-estimator to fail (see section 3.1).

The third option, noconstant, states that no constant term has to be considered in the regression. The fourth option, outlier, provides an estimate of robust standardized residuals, and of robust Mahalanobis distances. These can be used to construct a diagnostic plot, as discussed in Section 3.2, and the option graph calls on this graphical tool for outliers identification. Note that if there is not at least one continuous variable among the explanatory, an error message is returned. The robust standardized residuals are nevertheless computed. The option replic allows to fix the number of $p$ subsets to consider in the initial steps of the algorithm. The user can use equation (11) to change the value of $N$ in accordance to his desired level of $P_{\text {clean }}$ and/or $\alpha$. The default value for $N$ corresponds to $P_{\text {clean }}=0.99$ and $\alpha=0.2$. Finally, the option init will return as output the initial S-estimator, or the MS-estimator if the option dummy is invoked, instead of the final MM-estimator.

\section{Conclusion}

The strong impact of outliers on the least square regression estimator is known for a long time. Consequently, a large literature has been developed to find robust estimators that cope with the "atypical" observations, and have a high breakdown point. At the same time, the statistical efficiency of the robust estimators needs to remain sufficiently high. In recent years, it seems that a consensus has emerged to recommend the MM-estimators as the best suited estimation method, since they combine a high resistance to outliers and high efficiency at regression models with normal errors.

On the other hand, robust methods were not so often used by applied researchers, mainly because their practical implementation remained quite cumbersome. Over the last decade, efficient and relatively fast algorithms for computing robust estimators, including MM-estimators, were developed. Nowadays, the use of robust statistical methods becomes much more widespread in the applied sciences, like engineering and chemistry. By providing the Stata code, we make robust regression methods also available for the econometrics research community.

In this paper we summarize the properties of the best known robust estimation procedures and provide Stata code to implement them. We create the MMregress command (based on external functions that can be run separately). We furthermore show how this estimator outperforms all "robust" estimators available in Stata by mean of a modest simulation study. We hope that this paper will contribute to the development of further robust methods in Stata. In particular, development of robust 
procedures for Panel Data and time series models would be of major interest for applied economic research.

\section{References}

[1] Bramati, M. C. and C. Croux. 2007. Robust Estimators for the Fixed Effects Panel Data Model. Econometrics Journal 10(3): 521-540.

[2] Croux, C., G. Dhaene, and D. Hoorelbeke. 2008. Robust Standard Errors for Robust Estimators, manuscript. www.econ.kuleuven.be.christophe.croux/public.

[3] Edgeworth, F. Y. 1887. On Observations Relating to Several Quantities. Hermathena, 6: 279-285.

[4] Huber, P. 1964. Robust Estimation of a Location Parameter. Annals of Mathematical Statistics 35(1): 73-101.

[5] Huber, P. 1981. Robust Statistics. New York: John Wiley and Sons.

[6] Maronna, R., and V. 2006. Robust regression with both continuous and categorical predictors. Journal of Statistical Planning and Inference 89: 197-214.

[7] Maronna, R., D. Martin and V. 2006. Robust Statistics. New York: John Wiley and Sons.

[8] Rousseeuw, P. J.. Least Median of Squares Regression. Journal of the American Statistical Association 79: 871-880.

[9] Rousseeuw, P. J. and A. Leroy. 1987. Robust Regression and Outlier Detection. New York: John Wiley and Sons.

[10] Rousseeuw, P. J. and B. van Zomeren. 1990. Unmasking Multivariate Outliers and Leverage Points. Journal of the American Statistical Association 85: 633-639.

[11] Rousseeuw, P. J. and V. Yohai. 1987. Robust Regression by Means of S-estimators in Robust and Nonlinear Time Series Analysis: 256-272, edited by J. Franke, W. Härdle and D. Martin. Berlin: Springer Verlag.

[12] Salibian-Barrera, M. and V. Yohai. 2006. A Fast Algorithm for S-regression Estimates. Journal of Computational and Graphical Statistics 15: 414-427.

[13] Yohai, V. 1987. High Breakdown-point and High Efficiency Estimates for Regression. The Annals of Statistics 15: 642-665. 